\title{
Tradução, adaptação transcultural e validação de questionário de satisfação em telemedicina
}

\author{
Translation, cross-cultural adaptation and validation of a telemedicine satisfaction questionnaire \\ Traducción, adaptación transcultural y validación de cuestionario de satisfacción en \\ telemedicina
}

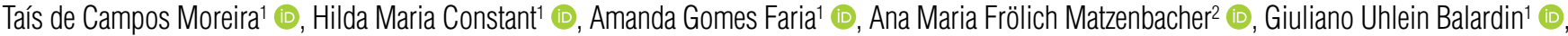

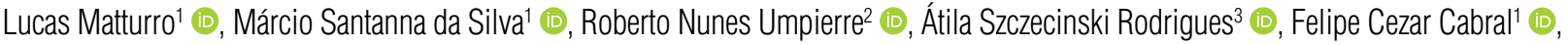 \\ Cassia Garcia Moraes Pagano ${ }^{1}$ (1)
}

${ }^{1}$ Hospital Moinhos de Vento - Porto Alegre (RS), Brasil.

2Universidade Federal do Rio Grande do Sul - Porto Alegre (RS), Brasil.

${ }^{3}$ Departamento de Saúde Digital, Secretaria Executiva, Ministério da Saúde - Brasília (DF), Brasil.

\section{Resumo}

Introdução: A telemedicina facilita o acesso ao cuidado para os pacientes. Essa tecnologia tem apresentado bons resultados clínicos e de satisfação dos usuários. A satisfação é um dos principais indicadores de qualidade dos serviços, e sua avaliação permite mudanças na qualidade da prestação de cuidados, identifica problemas e viabiliza a melhor gestão e os melhores comportamentos dos profissionais de saúde. Do aumento do uso da telemedicina no mundo emergiu a necessidade de entendimento da qualidade desse serviço. Objetivo: Traduzir, adaptar culturalmente e validar um questionário para avaliação da satisfação de pacientes atendidos por telemedicina. Métodos: A versão adaptada após a avaliação por um comitê de juízes foi utilizada em pré-teste com 30 pacientes atendidos no projeto TeleOftalmo. Os resultados do pré-teste foram avaliados a fim de se obter uma versão adequada do instrumento. Além disso, o instrumento foi aplicado em uma amostra de 141 pacientes atendidos via telemedicina. Análises de consistência interna e de validação de constructo foram realizadas. Resultados: O coeficiente de validade de conteúdo (CVC) global foi 0,942, demonstrando clareza, pertinência e relevância das questões. O instrumento apresentou consistência interna com alfa de Cronbach estandardizado de 0,6, considerado aceitável. A análise fatorial exploratória apresentou critério de Kaiser-Meyer-Olkin de adequação de amostragem de 0,56 e o teste de esfericidade de Bartlett apresentou valor de 0,001. Conclusões: A versão brasileira do Questionário de Avaliação da Satisfação de Pacientes Atendidos via Telemedicina (QAS-Tele) é um instrumento fácil e viável para a avaliação da satisfação dos pacientes atendidos por telemedicina.

Palavras-chave: Satisfação do paciente. Telemedicina. Estudos de validação. Inquéritos e questionários.

Como citar: Moreira TC, Constant HM, Faria AG, Matzenbacher AMF, Balardin GU, Matturro L, Silva MS, Umpierre RN Rodrigues AS, Cabral FC, Pagano CGM. Tradução, adaptação transcultural e validação de questionário de satisfação em telemedicina. Rev Bras Med Fam Comunidade. 2022;17(44):2837. https://doi.org/10.5712/rbmfc17(44)2837

\author{
Autor correspondente: \\ Amanda Gomes Faria \\ E-mail: amanda.facfar@gmail.com \\ Fonte de financiamento: \\ não se aplica \\ Parecer CAAE: \\ 16316919.1.0000.5330 \\ Procedência: \\ não encomendado. \\ Avaliação por pares: \\ externa. \\ Recebido em: 15/12/2020. \\ Aprovado em: 20/08/2021. \\ Editor Associado:
}

Francisco Eduardo da Fonseca Delgado 


\begin{abstract}
Introduction: Telemedicine facilitates the care in health by distance. This health technology has shown good clinical results and user satisfaction. The satisfaction is a main indication of service quality, your evaluation allows changes in the quality of care, identifies problems and enables better management and behaviors of health professionals. The increase in the use of telemedicine in the world has emerged in a need to understand the quality of this service. Objective: To translate, culturally adapt and validate a questionnaire to assess the satisfaction of patients treated by telemedicine. Methods: The version adapted after evaluation by a committee of judges was used in a pre-test with 30 patients seen in the project. The results of the pre-test were evaluated in order to obtain an adequate version of the instrument. In addition, this questionnaire was applied to a sample of 141 patients treated via telemedicine. Analysis of internal consistency and construct validation were performed. Results: The Global Content Validity Coefficient (CVC) score was 0.942 , demonstrating the questions' clarity, relevance and relevance. The instrument showed internal consistency with a standardized Cronbach's alpha of 0.6; considered acceptable. The exploratory factor analysis showed a KMO of 0.56 and Bartlett's sphericity test showed a value of 0.001. Conclusions: The Brazilian version of the Questionnaire for the Evaluation of Patient Satisfaction Via Telemedicine (QAS-Tele) is an easy and viable instrument for the evaluation of the satisfaction of patients treated by telemedicine.
\end{abstract}

Keywords: Patient satisfaction. Telemedicine. Validation study. Surveys and questionnaires.

\title{
Resumen
}

Introducción: La telemedicina facilita el acceso a la atención a los pacientes. Esta tecnología ha demostrado buenos resultados clínicos y en la satisfacción de los usuarios. La satisfacción es uno de los principales indicativos de la calidad de los servicios, su evaluación permite cambios en la calidad de la atención, identifica problemas y posibilita mejor gestión y comportamiento de los profesionales. El aumento del uso de la telemedicina en el mundo ha sugerido el entendimiento de la cualidad de esos servicios. Objetivo: Traducir, adaptar culturalmente y validar un cuestionario para evaluación de la satisfacción de pacientes atendidos por telemedicina. Métodos: Se desarrolló una evaluación por un comité de jueces con la versión adaptada y después se llevó a cabo un pre-test con 30 pacientes atendidos en el proyecto TeleOftalmo. La prueba previa ocurrió para obtener una versión adecuada del instrumento. Además, se aplicó en una muestra de 141 pacientes atendidos vía telemedicina. Análisis de consistencia interna y de validación de constructo fueron realizadas. Resultados: El coeficiente de validez de contenido (CVC) Global de 0,942, demostrando claridad, pertinencia y relevancia de las cuestiones. El instrumento presentó consistencia interna con alfa de Cronbach estandarizado de 0,6; considerado aceptable. El análisis factorial exploratorio presentó un KMO de 0,56 la prueba de esfericidad de Bartlett presentó valor de 0,001. Conclusiones: El Cuestionario Brasileño de Evaluación de la Satisfacción de los Pacientes Atendidos por Telemedicina (QAS-Tele) es fácil y viable para evaluar la satisfacción de los pacientes atendidos por telemedicina.

Palabras-clave: Satisfacción del paciente. Telemedicina. Estudio de validación. Encuestas y cuestionarios.

\section{INTRODUÇÃO}

A telessaúde é uma forma de prestar assistência em saúde utilizando tecnologias de informação e telecomunicação de forma ampla. A telemedicina, por sua vez, é mais específica e atua para dar suporte ao diagnóstico remoto por meio de laudos médicos a distância e segunda opinião qualificada. ${ }^{1} \mathrm{~A}$ telemedicina possibilita que os profissionais de saúde atuem a distância, facilitando o acesso ao cuidado para os pacientes. ${ }^{2,3} \mathrm{Em}$ períodos em que o distanciamento social é uma recomendação, como a pandemia do SARS-CoV-2, a telemedicina mostra-se uma ferramenta indispensável em resposta clínica à COVID-19, e a satisfação do paciente faz toda a diferença na resposta e na adesão ao tratamento. ${ }^{4}$

As consultas realizadas por telemedicina tendem a apresentar resultados clínicos e de satisfação dos pacientes semelhantes aos das consultas presenciais, ${ }^{5,6} \mathrm{com}$ a vantagem de ter acesso facilitado. ${ }^{3,4}$ Em um estudo realizado com pacientes atendidos por telemedicina nos Estados Unidos, 95\% dos entrevistados declararam-se muito satisfeitos com o cuidado que receberam nesse modelo de atendimento, demonstrando níveis de satisfação semelhantes aos obtidos para o atendimento presencial. ${ }^{7,8}$

A avaliação da satisfação é um indicador muito importante na saúde, seja no atendimento presencial, seja naquele por telemedicina. Trata-se de um conceito complexo, afetado por muitos fatores. ${ }^{9}$ Quanto aos componentes da satisfação, é possível avaliar o atendimento prestado pelos profissionais, a infraestrutura física, o tratamento recebido, a equidade no acesso ao diagnóstico, as terapias e medidas preventivas, a abordagem e a decisão nos procedimentos, o custo acessível, as informações adequadas, além do tempo de espera para o atendimento. ${ }^{10} \mathrm{Na}$ telemedicina, outros componentes também podem ser avaliados, como a qualidade da conexão, os equipamentos utilizados e a comunicação entre o profissional e o paciente. ${ }^{11}$ 
No entanto, as avaliações de satisfação por telemedicina tendem a ser realizadas por questionários breves, quantitativos, genéricos, não padronizados e não validados para esse contexto, escondendo as insatisfações dos pacientes que muitas vezes não podem pontuar as experiências negativas com alguns componentes da telemedicina, como por exemplo a forma de interação e as dificuldades com a tecnologia. ${ }^{12-14}$

Para a avaliação de qualquer desfecho, incluindo a satisfação, a validação de um questionário é uma etapa essencial para o seu uso. ${ }^{15} \mathrm{Em}$ relação à satisfação em telemedicina, o instrumento deve levar em consideração importantes aspectos dessa modalidade de atendimento, tais como experiência com o uso da tecnologia, conveniência para o paciente (em termos de tempo e economia com deslocamento), relacionamento e capacidade de comunicação médico-paciente, para que ele esteja adequado à avaliação da satisfação. ${ }^{15,16}$ Além disso, devem ser consideradas análises estatísticas que validem a confiabilidade do instrumento levando em consideração uma avaliação mais ampla da telemedicina, com a inclusão das diferentes modalidades do atendimento, em vez de questões genéricas sobre a satisfação. ${ }^{15}$

Apesar de existirem estudos relacionados à satisfação de pacientes em serviços de telemedicina em outros países, ${ }^{3,15}$ ainda são escassas as pesquisas que evidenciam os resultados de satisfação de pacientes atendidos por essa tecnologia no Brasil. Além disso, questionários consistentes em língua portuguesa para medir a satisfação nos atendimentos por telemedicina e indicar a qualidade da assistência e do serviço prestado não foram encontrados. Dessa forma, o objetivo deste estudo foi traduzir, adaptar culturalmente e validar o Questionário para Avaliação da Satisfação de Pacientes Atendidos via Telemedicina (QASTele) para avaliar a satisfação de pacientes atendidos por essa modalidade de atendimento no Brasil.

\section{MÉTODOS}

Trata-se de um estudo metodológico de tradução, adaptação e validação de um questionário para utilização no português brasileiro. O processo de validação da versão em português brasileiro do QASTele foi precedido pela solicitação de autorização para a utilização do instrumento ao autor principal. O questionário original, desenvolvido nos Estados Unidos por Hanna et al., ${ }^{17}$ possui 14 questões que abordam a satisfação geral, a experiência do paciente ao utilizar a telemedicina, a conveniência para o paciente, a relação médico-paciente e a capacidade de comunicação via telemedicina. As respostas são em escala Likert de 5 pontos, em que:

1 = "não, definitivamente não";

2 = "provavelmente não";

$3=$ "talvez";

4 = "provavelmente sim";

5 = "sim, com certeza".

A pontuação final do instrumento foi calculada utilizando-se a média das pontuações. O resultado é avaliado pela média numérica de todos os itens. Esse questionário foi escolhido para ser adaptado para o português brasileiro uma vez que é breve, de fácil aplicação e foi construído, em sua versão original, levando em consideração os princípios de confiabilidade, validade e generalização. ${ }^{18}$ Além disso, embora o questionário original use a palavra telessaúde em algumas afirmações, na prática foi aplicado num serviço de telemedicina.

A tradução e a adaptação cultural foram realizadas de acordo com a metodologia padrão. ${ }^{19}$ Procedeu-se à tradução direta para o português por dois tradutores bilíngues independentes (TM e RF). Em seguida, houve uma síntese das duas traduções, os tradutores discutiram as diferenças e chegaram a uma versão. A retradução 
realizada por dois nativos de língua inglesa com fluência na língua portuguesa (GF e SC) foi seguida pela consolidação realizada por um comitê de especialistas multidisciplinares que incluiu uma pedagoga (MP), uma especialista em validação de questionários de saúde $(\mathrm{HM})$ e uma enfermeira especialista em atendimentos por telemedicina (AM). Obteve-se assim uma versão consolidada, que foi submetida a validação de conteúdo com análise do coeficiente de validade de conteúdo (CVC). Esse cálculo levou em consideração a análise de quatro juízes (AMM, FCC, HMM, MCM) quanto à clareza de linguagem, à pertinência, à relevância teórica e à dimensão teórica. Os itens para a validade de conteúdo foram classificados numa escala Likert de 1 a 5, em que:

1 = pouquíssima;

2 = pouca;

3 = média;

4 = muita; $\mathrm{e}$

$5=$ muitíssima. $^{19}$

Para o processo de validação, foram incluídos no estudo os pacientes usuários do Sistema Único de Saúde (SUS), acima de 18 anos, que aceitaram participar da pesquisa quando chegaram para o telediagnóstico no TeleOftalmo. ${ }^{20} \mathrm{O}$ TeleOftalmo é um projeto no qual foram implementados oito consultórios oftalmológicos remotos em macrorregiões de saúde do estado do Rio Grande do Sul para realizar testes oftalmológicos coordenados a distância por oftalmologistas e localmente acompanhados por uma equipe de enfermagem treinada previamente. Os pacientes são incluídos numa plataforma pelos médicos da atenção primária e do SUS e depois agendados para o atendimento. ${ }^{20}$ Foram excluídos do estudo aqueles pacientes com dificuldades cognitivas, aferidas pelas suas dificuldades em compreender onde estava e por quais motivos estavam no local do telediagnóstico, bem como os que não passaram pela etapa síncrona de atendimento com o oftalmologista.

A versão adaptada foi utilizada no pré-teste, aplicado em 30 pacientes atendidos no ponto remoto localizado na macrorregião metropolitana de saúde. A maioria era do sexo feminino $(57 \%)$, com média de idade de 49 anos e ensino fundamental incompleto (46\%). Esses pacientes não foram incluídos na etapa seguinte do processo de validação do questionário.

Seguindo as etapas do processo, realizou-se então a validação semântica. O objetivo dessa etapa foi verificar a compreensão dos itens pela população a que o instrumento se destina. Nela, realizou-se a avaliação de compreensão do questionário, e as perguntas que não foram compreendidas pelos pacientes passaram por revisão e modificações. Para ajuste na expressão do instrumento foi criado um grupo de consolidadores, composto de profissionais da saúde expertos na área de telemedicina (TM,CP e FC), que realizaram reuniões nas quais foram discutidas medidas para facilitar o entendimento das perguntas por parte dos usuários. $O$ critério de seleção desses profissionais deu-se com base em sua experiência com telemedicina, validação de instrumentos e aplicação de instrumentos na população testada. A Tabela 1 apresenta o questionário com as versões original, traduzida, adaptada, retraduzida e final.

A equipe de enfermagem foi treinada para a aplicação do instrumento com aulas expositivas e discussão para os profissionais participantes. Foram realizadas sessões conjuntas de treinamento na aplicação do questionário, em que cada entrevistador treinava com o colega e os resultados eram discutidos posteriormente para avaliar a técnica e corrigir possíveis erros de coleta. As sessões foram as seguintes:

1. O entrevistador foi orientado a acolher o usuário, explicar o estudo, obter sua anuência para a participação com a assinatura do Termo de Consentimento Livre e Esclarecido (TCLE) e explicar qualquer dúvida;

2. Foram coletados os dados sociodemográficos, como idade, escolaridade e renda;

3. O entrevistador realizou a leitura de cada questão da versão adaptada do instrumento. 
Tabela 1. Questionário para avaliação da satisfação em telemedicina nas suas versões original, traduzida, adaptada, retraduzida e final. Porto Alegre, Brasil, 2020.

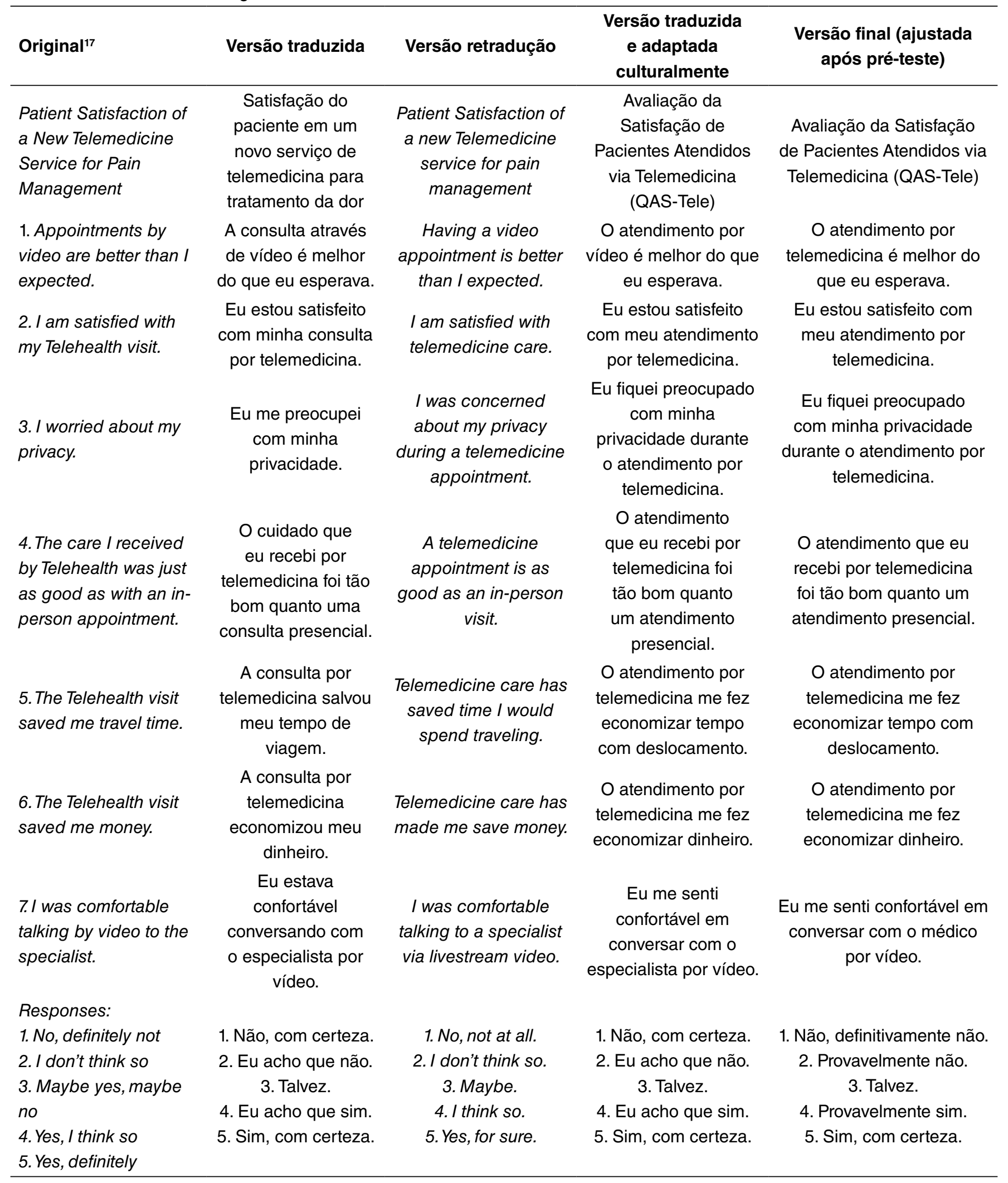


Tabela 1. Continuação.

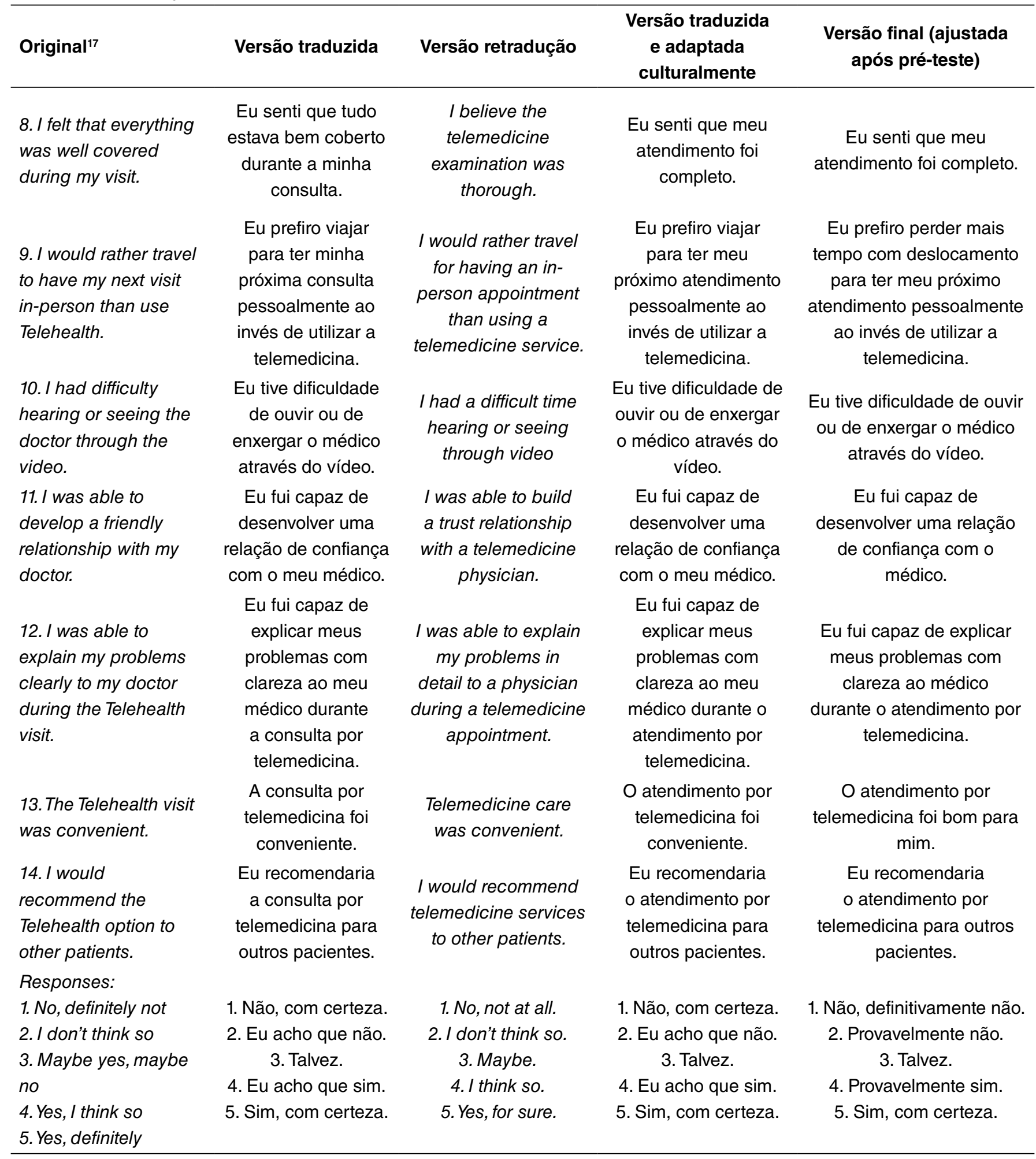

Para o tamanho da amostra, considerou-se que para o processo de validação do questionário se recomenda a amostra de dez pacientes para cada pergunta do questionário a ser validado. ${ }^{19,21}$ Assim como o QAS-Tele é composto de 14 questões, foram entrevistados 141 pacientes, e a amostragem foi aleatória simples.

Dos 163 convidados, 23 recusaram-se a participar por falta de tempo para responder ao questionário; assim, a versão final foi aplicada em 141 pacientes atendidos pelo projeto TeleOftalmo. Todos os pacientes 
atendidos no período foram convidados a participar do estudo, no entanto esse foi um período com menos pacientes agendados e com absenteísmo elevado em razão da pandemia. O projeto, no ano de 2020, atendeu em torno de 100 pacientes/mês no consultório onde foi aplicado o questionário. As respostas obtidas foram registradas por meio do questionário implementado, com as seguintes seções:

1. Acolhimento ao usuário e aplicação do TCLE;

2. Dados sociodemográficos - ao perguntarem qual a renda familiar do participante, os coletadores explicaram o valor de referência do salário-mínimo;

3. Questões do instrumento traduzido e adaptado.

Realizou-se análise descritiva dos dados para caracterizar a amostra, e as variáveis quantitativas foram descritas na forma de média e desvio padrão, enquanto as variáveis categóricas foram apresentadas como frequência absoluta e relativa. O CVC foi calculado considerando-se aceitáveis as questões com valor $>0,80 .{ }^{19} \mathrm{Um}$ teste de Kappa foi executado para verificar a concordância entre a avaliação dos quatro juízes, e os seguintes critérios foram utilizados:

$<0$ = discordância;

$0-0,20$ = quase nenhuma;

$0,20-0,40$ = pequena;

$0,40-0,60=$ moderada;

$0,60-0,80=$ substancial;

$0,80-1,00=$ quase perfeita. ${ }^{19}$

O coeficiente alfa de Cronbach (consistência interna) foi calculado como medida de concordância entre as médias dos itens do instrumento. Consideraram-se valores do alfa de Cronbach iguais ou maiores que 0,6 como indicativos de consistência interna no questionário. ${ }^{22}$

A validade de construto foi realizada por análise fatorial exploratória (AFE). Para verificar a adequação da análise fatorial, foi utilizado o índice de Kaiser-Meyer-Olkin Measure of Sampling Adequacy (KMO) e o teste de esfericidade de Bartlett $(p<0,05)$, que avalia a presença de correlações entre as variáveis. Os dados foram coletados em uma planilha eletrônica de dados, importada para análise no software Statistical Package for the Social Sciences (SPSS) Inc. Released 2009, Predictive Analytics Software (PASW) for Windows, version 21.0 (Chicago: SPSS Inc). O nível de significância adotado para a inferência estatística foi de 5,0\%. O projeto foi aprovado pelo Comitê de Ética do Hospital Moinhos de Vento (número do parecer 3.466.154).

\section{RESULTADOS}

As análises realizadas contribuíram para a consolidação da versão do QAS-Tele em português brasileiro. $\mathrm{Na}$ etapa de tradução e adaptação transcultural a análise dos escores obtidos dos avaliadores (CVC) foi de 0,942 na avaliação global, nas análises por questão todos os valores de CVC foram maiores que 0,8, demonstrando a clareza, a pertinência e a relevância das questões. Em relação às dimensões satisfação geral, experiência do paciente ao utilizar a telemedicina, conveniência para o paciente, relação médico-paciente e capacidade de comunicação via telemedicina, foi encontrado um percentual de concordância entre os juízes de $65 \%$ e índice Kappa de 0,57 (intervalo de confiança - IC95\% 0,35-0,78), considerado moderado.

A versão adaptada foi aplicada no pré-teste em uma amostra de 30 usuários do projeto e observouse uma dificuldade dos pacientes em compreender as questões 8, 9 e 13. Estas foram alteradas para 
ficarem culturalmente mais fáceis aos participantes falantes de português brasileiro, conforme apresenta a Tabela 1.

Aversão adaptada foi aplicada em 141 pacientes atendidos em um serviço de diagnóstico oftalmológico a distância. Entre os entrevistados, 95 (67\%) eram mulheres, com média de idade de 53 anos (DP \pm 15$) ; 50$ $(35,5 \%)$ tinham ensino fundamental incompleto; 43 (30,5\%) apresentavam renda mensal de um saláriomínimo; a média de tempo de espera para o atendimento diagnóstico a distância foi de 224 dias. A Tabela 2 apresenta as principais características sociodemográficas dos participantes do estudo.

Tabela 2. Características sociodemográficas dos pacientes atendidos no serviço de teleoftalmologia que responderam ao Questionário de Avaliação da Satisfação de Pacientes Atendidos via Telemedicina $(n=141)$. Porto Alegre, Brasil, 2020.

\begin{tabular}{|c|c|c|}
\hline Características dos pacientes & $\mathbf{n}$ & $\%$ \\
\hline \multicolumn{3}{|l|}{ Sexo } \\
\hline Feminino & 95 & 67,4 \\
\hline Masculino & 46 & 32,6 \\
\hline \multicolumn{3}{|l|}{ Escolaridade } \\
\hline Analfabeto & 7 & 4,9 \\
\hline Fundamental incompleto & 50 & 35,5 \\
\hline Fundamental completo & 29 & 20,6 \\
\hline Médio completo & 47 & 33,3 \\
\hline Superior & 8 & 5,7 \\
\hline \multicolumn{3}{|l|}{ Renda familiar } \\
\hline Menos de 1 salário-mínimo (menos de $\mathrm{R} \$ 998,00$ ) & 16 & 11,3 \\
\hline De 1 salário-mínimo $(R \$ 998,00)$ até dois (até $R \$ 1.996,00)$ & 86 & 61,0 \\
\hline$\geq 3$ salários-mínimos $(\geq R \$ 2.994,00)$ & 39 & 27,7 \\
\hline \multicolumn{3}{|l|}{ Raça autorreferida } \\
\hline Branca & 90 & 63,8 \\
\hline Preta & 25 & 17,7 \\
\hline Parda & 21 & 14,9 \\
\hline Outros & 5 & 3,6 \\
\hline Idade (média; $\pm \mathrm{DP}$ ) & 53,4 & 15,3 \\
\hline Total & 141 & 100 \\
\hline
\end{tabular}

DP: desvio padrão.

As médias para as respostas dos 141 pacientes atendidos via telemedicina, para cada uma das questões do instrumento, são apresentadas na Tabela 3. As questões 3, 9 e 10 são afirmações negativas; dessa forma, as respostas tiveram pontuação em disposição invertida, apenas para fins de análise. As demais questões são afirmações positivas.

$\mathrm{Na}$ avaliação da consistência interna o alfa de Cronbach estandardizado foi de 0,6, mostrandose aceitável nesse quesito..22,23 Optou-se pelo alfa de Cronbach estandardizado por ele representar adequadamente a homogeneidade dos itens do instrumento. ${ }^{24} \mathrm{~A}$ análise fatorial exploratória apresentou o critério de Kaiser-Meyer-Olkin de adequação de amostragem (KMO) de 0,56; por ser um valor considerado inaceitável, não se passou para a análise fatorial confirmatória. Por outro lado, o teste de esfericidade de Bartlett apresentou valor de 0,001, demonstrando que, com base nesse índice, existia a possibilidade de 
Tabela 3. Médias de resposta dos 141 pacientes atendidos via telemedicina para cada uma das questões do questionário de satisfação em validação. Porto Alegre, Brasil, 2020.

\begin{tabular}{llc}
\hline Questão & Média & \pm DP \\
\hline 1. O atendimento por telemedicina é melhor do que eu esperava & 4,88 & 0,36 \\
2. Eu estou satisfeito com meu atendimento por telemedicina & 4,90 & 0,32 \\
3. Eu fiquei preocupado com minha privacidade durante o atendimento por telemedicina & 4,87 & 0,51 \\
4. O atendimento que eu recebi por telemedicina foi tão bom quanto um atendimento presencial & 4,83 & 0,47 \\
5. O atendimento por telemedicina me fez economizar tempo com deslocamento & 3,84 & 1,60 \\
6. O atendimento por telemedicina me fez economizar dinheiro & 4,60 & 1,01 \\
7. Eu me senti confortável em conversar com o médico por vídeo & 4,89 & 0,54 \\
8. Eu senti que meu atendimento foi completo & 4,94 & 0,37 \\
9. Eu prefiro perder mais tempo com deslocamento para ter meu próximo atendimento & 4,56 & 1,05 \\
pessoalmente ao invés de utilizar a telemedicina & 4,73 & 0,89 \\
10. Eu tive dificuldade de ouvir ou de enxergar o médico através do vídeo & 4,80 & 0,57 \\
11. Eu fui capaz de desenvolver uma relação de confiança com o médico & 4,80 & 0,57 \\
12. Eu fui capaz de explicar meus problemas com clareza ao médico durante o & 4,97 & 0,14 \\
atendimento por telemedicina & 4,98 & 0,11 \\
13. O atendimento por telemedicina foi bom para mim & & \\
14. Eu recomendaria o atendimento por telemedicina para outros pacientes
\end{tabular}

DP: desvio padrão.

fatoração do instrumento, a qual foi inviabilizada em função da não uniformidade das medidas (KMO e Bartlett). ${ }^{25}$ Optou-se, portanto, pelo modelo unidimensional (1-fator).

\section{DISCUSSÃO}

O objetivo deste estudo foi traduzir, adaptar culturalmente e validar um questionário para a avaliação da satisfação de pacientes atendidos por telemedicina no Brasil. A avaliação de equivalência conceitual e dos itens do QAS-Tele demonstrou que o instrumento é viável e de fácil aplicação para ser utilizado na avaliação da satisfação de pacientes atendidos por telemedicina. No questionário original em inglês essa validação não havia sido realizada. A equivalência conceitual procurou explorar o construto de interesse (satisfação) na sua definição original e na cultura brasileira. As etapas de tradução e retradução da versão original do instrumento foram criteriosas. Houve atenção especial para não usar expressões com influências regionais ou gírias. A validação de conteúdo foi considerada adequada porque todos os escores (CVC) encontrados, tanto o escore global quanto o individual de cada questão, foram superiores a $0,80 .{ }^{19}$ A validação do conteúdo é etapa essencial neste tipo de estudo, pois permite verificar se os itens do questionário representam adequadamente o que é necessário ser medido - neste caso a relevância teórica para a avaliação da satisfação dos pacientes atendidos por telemedicina. ${ }^{26} \mathrm{O}$ estudo original de Hanna et al. ${ }^{17}$ não executou essa análise, de modo que não foi possível realizar uma comparação dessa fase entre o instrumento original e o adaptado para o português.

Mair e Whitten, ${ }^{27}$ em seu estudo, revisaram pesquisas sobre a satisfação do paciente em teleatendimento. Nos estudos encontrados, a grande maioria utilizou instrumentos de levantamento simples e as metodologias utilizadas para avaliar a satisfação não foram claramente especificadas, o que inviabiliza a comparação dos dados. Além disso, poucos deles definiram o que significava 
satisfação e apresentavam apenas impressões iniciais. Em nosso trabalho demonstramos que a metodologia está detalhadamente descrita, e sugerimos que sempre que o instrumento for aplicado sua avaliação de confiabilidade seja realizada para determinar como ele se comportou na população aplicada. Além disso, os aplicadores foram orientados a explicar o conceito de satisfação. Embora o instrumento não tenha diferentes dimensões, contempla a avaliação da satisfação do paciente para além das impressões iniciais. As questões abordam diferentes aspectos, como a experiência dos pacientes com a telemedicina, a conveniência do atendimento, as preocupações com a privacidade e se eles recomendariam tal atendimento.

No estudo original de Hanna et al., ${ }^{17}$ não fica claro como o questionário foi respondido pelos participantes, se por entrevista face a face ou se ele foi autoadministrado. A aplicação do instrumento por meio de entrevistas face a face era a opção mais adequada em nosso meio, em virtude das características da população atendida, com dificuldades visuais e menor grau de instrução. A avaliação da compreensão no estudo pré-teste mostrou que o instrumento foi adequado para ser aplicado por um entrevistador, facilitando a compreensão dos entrevistados, especialmente por se tratar de uma amostra de pessoas com problemas de visão e de baixa escolaridade.

De forma geral, a média para as 14 questões do questionário foi elevada, indicando bom nível de satisfação. Outras avaliações de satisfação realizadas em serviços de saúde do SUS que não contemplam a telemedicina mostram que há uma avaliação positiva da rede pública no Brasil, no entanto, atreladas a isso, existem inúmeras dificuldades apontadas pelos pacientes e relacionadas ao atendimento, ao longo tempo de espera e às filas, à deficiência de recursos físicos e materiais. ${ }^{28}$ Durante o processo de validação, foi observado que algumas questões não eram de fácil entendimento para os pacientes, provavelmente pelo fato de o instrumento ser aplicado num projeto no âmbito do SUS e em virtude das condições sociodemográficas. Assim, as questões 5 e 6 - " $O$ atendimento por telemedicina me fez economizar tempo com deslocamento" e "O atendimento por telemedicina me fez economizar dinheiro" - deixavam alguns pacientes confusos. Isso porque os pacientes sempre tinham que se deslocar ao local mais próximo de sua residência para a realização dos exames e esses são gratuitos para os usuários do SUS. Sendo assim, dependendo da realidade em que será aplicado o instrumento, essas são questões que devem ser explicadas com maiores detalhes pelo entrevistador. Esse é um ponto importante a se considerar no treinamento dos profissionais para aplicação do questionário.

A análise de consistência interna forneceu valor do alfa de Cronbach estandardizado de 0,6 - valor aceitável, conforme indicam Ursachi et al. ${ }^{22} \mathrm{O}$ alfa tende a ser modificado pelo tamanho do instrumento, e em testes pequenos se apresenta reduzido. ${ }^{22}$ Além disso é uma estimativa, e indicase que toda vez que o questionário for aplicado calcule-se um novo alfa uma vez que essa medida pode sofrer alteração. ${ }^{22}$ Algumas testagens para retiradas de questões foram realizadas, no entanto as tentativas seguindo pressupostos estabelecidos diminuíram o valor do alfa. Sendo assim, o melhor formato do questionário foi o que contemplava todas as questões conforme o original.

No processo da avaliação fatorial exploratória foi confirmada a estrutura de 14 itens, sem a indicação de retirada de questões ou determinação de diferentes dimensões. Isso corrobora a avaliação da concordância moderada entre os juízes, fato que pode ser explicado em razão de algumas questões terem correspondência em mais de uma das dimensões, sendo difícil, dessa forma, a distribuição adequada das questões entre as diferentes dimensões sugeridas pelo autor da versão original. Por exemplo, "Eu me senti confortável em conversar com o médico por vídeo" pode corresponder à 
dimensão "experiência do paciente" e também à "capacidade de comunicação via telemedicina". Na testagem da inserção das dimensões sugeridas pelo autor da versão original do instrumento (satisfação geral, experiência do paciente ao utilizar a telemedicina, conveniência para o paciente, relação médicopaciente e capacidade de comunicação via telemedicina), observou-se na análise estatística a não uniformidade dos índices KMO e Bartlett na versão de língua portuguesa, optando-se pela estrutura do instrumento sem dimensões. ${ }^{24} \mathrm{Na}$ versão original construída por Hanna et al. ${ }^{17}$ não houve avaliação fatorial, embora o autor tenha sugerido o uso das dimensões e testado o questionário.

Algumas limitações devem ser destacadas em relação a este questionário e à sua aplicação.

a) Muitos pacientes desta amostra esperaram mais de 220 dias para terem atendimento especializado, o que pode ter modificado a percepção da satisfação. Talvez esses pacientes se tenham sentido gratos pela oportunidade de consultar um oftalmologista e não tenham conseguido de verdade avaliar os itens da satisfação questionados.

b) Todos os pacientes são usuários do SUS, e comparações com amostras de serviços privados não puderam ser realizadas, pois no Brasil o modelo de atendimento por telemedicina teve seu início durante a pandemia da COVID-19 e portanto não havia publicações com esse tema quando este estudo foi finalizado.

c) A satisfação é uma questão complexa afetada por muitos fatores, ${ }^{9}$ de modo que seria importante a aplicação do questionário em diferentes cenários a fim de se buscar o melhor entendimento a respeito dos componentes da satisfação abordados no instrumento. Além disso, o questionário foi validado na região Sul do Brasil e pode não representar todas as características da população brasileira.

d) Embora a consistência interna do instrumento tenha sido aceitável, espera-se que novos resultados demonstrem consistência interna muito boa. Além disso, a opção do modelo unidimensional poderá ser melhor explorada.

É importante ressaltar a necessidade de realização de novos estudos para avaliar a validade e a confiabilidade do QAS-Tele utilizando amostras representativas de outros serviços de telemedicina. Principalmente neste momento em que o mundo passou a fazer um maior uso da telemedicina, um processo contínuo de avaliação do instrumento deve ser realizado para aprimorar a validade de construto do questionário. Apesar de o instrumento de estudo usar o conceito de telemedicina, ele é aplicável aos serviços de telessaúde uma vez que estes preveem maior número de iniciativas.

A versão brasileira do QAS-Tele pode ser disponibilizada para aplicação em serviços de telessaúde brasileiros. Salienta-se que a avaliação da confiabilidade de qualquer instrumento deve ocorrer sempre para a verificação de como o instrumento irá se comportar em determinada população. Novos estudos podem vir a contribuir para a avaliação de como o instrumento se comporta em diferentes regiões do país e a confirmação da unidimensionalidade.

Este trabalho disponibiliza um instrumento fácil e viável de avaliação da satisfação dos pacientes atendidos por telemedicina, a ser aplicado por um entrevistador. A telemedicina no Brasil, durante a pandemia, modificou-se completamente; assim, instrumentos para avaliação da satisfação de pacientes e de profissionais envolvidos nesse modelo de atendimento devem ser criados e validados para auxiliar na qualificação do serviço prestado, garantindo a otimização do acesso à rede de saúde e a familiarização dos pacientes com esse novo formato de atendimento. 


\section{AGRADECIMENTOS}

Aos tradutores Rafael Michita e Atanásio Gaetano Fava e à pedagoga Marcela Paseto suas constribuições nas etapas de validação deste instrumento. À pesquisadora Maria Cristina Matte a avaliação do instrumento, aos técnicos de enfermagem Wagner Rosa, Marianny Souto, Luana Ribeiro, Juliana Assis, Ana Lopes e Daiana Michelline a aplicação do instrumento nos pacientes e ao Núcleo de Apoio à Pesquisa do Hospital Moinhos de Vento (NAP) o apoio em todo o projeto.

\section{CONFLITO DE INTERESSES}

Nada a declarar.

\section{CONTRIBUIÇÕES DOS AUTORES}

TCM: Administração do projeto, Análise formal, Conceituação, Escrita - primeira redação, Escrita - revisão e edição, Metodologia, Recursos, Software, Supervisão, Visualização. HMC: Análise formal, Metodologia, Recursos, Validação, Visualização. AGF: Curadoria de dados, Escrita - primeira redação, Investigação, Validação, Visualização. AMFM: Curadoria de dados, Escrita - primeira redação, Investigação, Validação, Visualização. GUB: Curadoria de dados, Escrita - primeira redação, Investigação, Validação, Visualização. LM: Curadoria de dados, Escrita - primeira redação, Investigação, Validação, Visualização. MSS: Curadoria de dados. RNU: Escrita - primeira redação, Investigação, Validação, Visualização. ASR: Escrita - primeira redação, Investigação, Validação, Visualização. FCC: Curadoria de dados, Escrita - primeira redação, Investigação, Obtenção de financiamento, Validação, Visualização. CGMP: Administração do projeto, Análise formal, Conceituação, Escrita - primeira redação, Escrita revisão e edição, Metodologia, Recursos, Software, Supervisão, Visualização.

\section{REFERÊNCIAS}

1. Silva AB. Telessaúde no Brasil - conceitos e aplicações. Rio de Janeiro: DOC; 2014.

2. Polinski JM, Barker T, Gagliano N, Sussman A, Brennan TA, Shrank WH. Patients' satisfaction with and preference for telehealth visits. J Gen Intern Med. 2016;31(3):269-75. https://doi.org/10.1007/s11606-015-3489-X

3. Kruse CS, Krowski N, Rodriguez B, Tran L, Vela J, Brooks M. Telehealth and patient satisfaction: a systematic review and narrative analysis. BMJ Open. 2017;3;7(8):e016242. https://doi.org/10.1136/bmjopen-2017-016242

4. Ramaswamy A, Yu M, Drangsholt S, Ng E, Culligan PJ, Schlegel PN, Hu JC. Patient satisfaction with telemedicine during the COVID-19 pandemic: retrospective cohort study. J Med Internet Res. 2020;22(9):e20786. https://doi.org/10.2196/20786

5. Campos PD, Ferrari DV. Teleaudiology: evaluation of teleconsultation efficacy for hearing aid fitting. J Soc Bras Fonoaudiol. 2012;24(4):301-8. https://doi.org/10.1590/s2179-64912012000400003

6. Sjöström M, Umefjord G, Stenlund H, Carlbring P, Andersson G, Samuelsson E. Internet-based treatment of stress urinary incontinence: 1- and 2-year results of a randomized controlled trial with a focus on pelvic floor muscle training. BJU Int. 2015;116(6):955-64. https://doi.org/10.1111/bju.13091

7. Nesbitt TS, Marcin JP, Daschbach MM, Cole SL. Perceptions of local health care quality in 7 rural communities with telemedicine. J Rural Health. 2005;21(1):79-85. https://doi.org/10.1111/j.1748-0361.2005.tb00066.x

8. Uscher-Pines L, Mehrotra A. Analysis of Teladoc use seems to indicate expanded access to care for patients. Health Aff (Millwood). 2014;33(2):258-64. https://doi.org/10.1377/hlthaff.2013.0989

9. Batbaatar E, Dorjdagva J, Luvsannyam A, Amenta P. Conceptualisation of patient satisfaction: a systematic narrative literature review. Perspect Public Health. 2015;135(5):243-50. https://doi.org/10.1177/1757913915594196

10. Mpinga EK, Chastonay P. Satisfaction of patients: a right to health indicator? Health Policy. 2011;100(2-3):144-50. https://doi. org/10.1016/j.healthpol.2010.11.001 
11. Dlugonski D, Motl RW, Mohr DC, Sandroff BM. Internet-delivered behavioral intervention to increase physical activity in persons with multiple sclerosis: sustainability and secondary outcomes. Psychol Health Med. 2012;17(6):636-51. https://doi. org/10.1080/13548506.2011.652640

12. Williams TL, May CR, Esmail A. Limitations of patient satisfaction studies in telehealthcare: a systematic review of the literature. Telemed J E Health. 2001;7(4):293-316. https://doi.org/10.1089/15305620152814700

13. Mair F, Whitten P. Systematic review of studies of patient satisfaction with telemedicine. BMJ. 2000;320(7248):1517-20. https://doi.org/10.1136/bmj.320.7248.1517

14. Collins K, Nicolson P, Bowns I. Patient satisfaction in telemedicine. Health Informatics Journal. 2000;6(2):81-5. https://doi. org/10.1177/146045820000600205

15. Bakken S, Grullon-Figueroa L, Izquierdo R, Lee NJ, Morin P, Palmas W, et al. Development, validation, and use of English and Spanish versions of the telemedicine satisfaction and usefulness questionnaire. J Am Med Inform Assoc. 2006;13(6):660-7. https://doi.org/10.1197/jamia.M2146

16. Gustke SS, Balch DC, West VL, Rogers LO. Patient satisfaction with telemedicine. Telemedicine Journal. 2000;6(1):5-13. https://doi.org/10.1089/107830200311806

17. Hanna GM, Fishman I, Edwards DA, Shen S, Kram C, Liu X, et al. Development and patient satisfaction of a new telemedicine service for pain management at Massachusetts General Hospital to the Island of Martha's Vineyard. Pain Med. 2016;17(9):1658-63. https://doi.org/10.1093/pm/pnw069

18. Demiris G. Principles of survey development for telemedicine applications. J Telemed Telecare. 2006;12(3):111-5. https://doi. org/10.1258/135763306776738549

19. Pasquali L. Instrumentação psicológica: fundamentos e prática. Porto Alegre: Artmed; 2010.

20. Araújo AL, Moreira TC, Rados DRV, Gross PB, Molina-Bastos CG, Katz N, et al. The use of telemedicine to support Brazilian primary care physicians in managing eye conditions: the TeleOftalmo Project. PLoS One. 2020;15(4):e0231034. https://doi. org/10.1371/journal.pone.0231034

21. Hair Jr JF, Black WC, Babin BJ, Anderson RE, Tatham RL. Análise multivariada de dados. 6a ed. Porto Alegre: Bookman; 2009.

22. Ursachi G, Horodnic IA, Zait A. How reliable are measurement scales? External factors with indirect influence on reliability estimators. Procedia Economics and Finance. 2015;20:679-86. https://doi.org/10.1016/S2212-5671(15)00123-9

23. DeVellis RF. Scale development: theory and applications. Newbury Park: SAGE Publications; 1991.

24. Maroco J, Garcia-Marques T. Qual a fiabilidade do alfa de Cronbach? Questões antigas e soluções modernas? Laboratório de Psicologia. 2006;4(1):65-90.

25. Damásio BF. Uso da análise fatorial exploratória em psicologia. Aval Psicol. 2012;11(2):213-28.

26. Alexandre NMC, Coluci MZO. Validade de conteúdo nos processos de construção e adaptação de instrumentos de medidas. Ciênc Saúde Coletiva. 2011;16(7):3061-8. https://doi.org/10.1590/S1413-81232011000800006

27. Mair F, Whitten P. Systematic review of studies of patient satisfaction with telemedicine. BMJ. 2000;320(7248):1517-20. https://doi.org/10.1136/bmj.320.7248.1517

28. Moimaz SAS, Marques JAM, Saliba O, Garbin CAS, Zina LG, Saliba NA. Satisfação e percepção do usuário do SUS sobre o serviço público de saúde. Physis. 2010;20(4):1419-40. https://doi.org/10.1590/S0103-73312010000400019 